Int. J. Environ. Res. Public Health 2008, 5(1), 1-3

International Journal of

Environmental Research and Public Health

ISSN 1661-7827

www.ijerph.org

(c) 2008 by MDPI

\title{
Editorial
}

\section{Fourth International Symposium on Recent Advances in Environmental Health Research}

\author{
Abdul K. Mohamed ${ }^{1}$, Mark G. Hardy², and Paul B. Tchounwou ${ }^{3 *}$ \\ ${ }^{1}$ Dean Emeritus and Director; ${ }^{2}$ Dean; ${ }^{3}$ Associate Dean and Deputy Director, National Institutes of Health RCMI-Center for \\ Environmental Health, College of Science, Engineering and Technology, Jackson State University, Jackson, MS 39217, USA. \\ *Correspondence to Dr. Paul B. Tchounwou; Email: paul.b.tchounwou@jsums.edu
}

The Fourth International Symposium on Recent Advances in Environmental Health Research was organized by Jackson State University (JSU) from September 16-19, 2007 at the Marriott Hotel in Jackson, Mississippi. The Symposium was built upon the overwhelming success of the previous symposia hosted by JSU and co-sponsored by the National Institutes of Health (NIH) RCMI-Center for Environmental Health, the U.S. Department of Education Title III Graduate Education Program, the U.S. Environmental Protection Agency, and the JSU office of Research.

In an attempt to contribute global solutions to major environmental health challenges, scientists around the world have been more and more involved in bioenvironmental research, studying the toxic mechanisms of action of various environmental agents, developing new approaches for detecting or remedying environmental damage, identifying and characterizing genes involved in the manifestation of environmentally-related diseases, and providing the public and policy makers with scientific tools that are critical for environmental and human health decision-making.

Building on the foundation of the previous symposia, the Fourth International Symposium on Recent Advances in Environmental Health Research served as a platform for environmental and biomedical scientists-biologists, chemists, toxicologists, public health scientists, engineers, and policy makers interested in bringing about substantial contributions to addressing global environmental and sustainable development issues, to communicate the latest advances in scientific research and new developments on critical environmental and human health topics. As in previous symposia, the Fourth Symposium focused on several important topics including the following:

- New Frontiers in Environmental Health Research: The causes of most human diseases have been attributed to the complex interactions between genetic factors and environmental exposures. Hence, control and prevention measures highly rely on the understanding of the cause and effect relationships between these factors and disease development. In recent years, new areas of research such as toxicogenomics, proteomics, and functional genomics have emerged, with the aim of understanding molecular mechanisms of health and disease. Also, the recent advances in the molecular biology of the cell cycle regulation have given new life to our understanding of cancer in particular, and the idea that defects of regulation in cancer cells may partially explain successes that have been achieved in cancer chemotherapy. Specific areas of symposium research presentations included gene expression studies, proteomics, gene-environment interactions, functional genomics, biomarkers of effect, sensitivity and effect, signal transduction and gene activation; and molecular targets of disease chemotherapy.

- Environmental Toxicology and Health Risk Assessment: Growing public awareness of the potential risk to humans from toxic chemicals in the environment has generated demand for new and improved methods for toxicity assessment and rational means for estimating health risk. Many environmental agents such as metal ions, polycyclic aromatic hydrocarbons, pesticides/herbicides, UV- 
light, food additives, and viruses are known to induce various types of illnesses including cancer in humans. Several symposium presentations dealt with research elucidating the cellular and molecular mechanisms by which these environmental agents induce toxicity, mutagenesis, and carcinogenesis, as well as research on hazard assessment of exposure to physical, chemical and biological agents; dose-response evaluation and model development; exposure assessment analysis; and health risk characterization; and management.

- Emerging Topics in Computational Biology, and Environmental Modeling: Using of computational methods and procedures to investigate environmental and biological phenomena has made remarkable progresses. This field includes analysis of human genome data, prediction of DNA and protein structure and function, design of biomaterials and therapeutic agents, studies into small molecule-biomacromolecule interactions, and other related computational method development. Therefore, several symposium presentations dealt with the computational analysis of the physical and chemical properties of several environmental compounds, as well as on quantitative structure activity relationship (QSAR) studies for developing predictive toxicology models associated with exposure to these compounds.

- Health Disparities and Environmental Security: In recent years health disparities and biological and chemical terrorism have emerged as major issues in public safety and homeland security. With recent advances in laboratory technologies, it is often possible to measure specific genetic variations as risk factors for specific types of disease. Equally important is the evaluation of the role of modifier factors such as environmental exposures or other genes that may exacerbate the genetic risk leading to differences in disease susceptibility among individuals. Since the events of September 11, 2001 regarding the attacks on the World Trade Center and the Pentagon, and the subsequent anthrax attacks on several people, our collective thinking with regard to our vulnerability to terrorism has completely changed. The specific areas of research presentations included the following: health disparities and cancer; health disparities and heart disease; health disparities and infectious diseases; and bioterrorism/chemical terrorism.

- Medical Geology and Human Health: Recent concerns over health-related issues arising from exposure to environmental substances have raised substantial interest in a new field termed "medical geology”. In fact, naturally occurring toxic metals such as arsenic, cadmium, lead, and mercury are now known to cause serious public health problems in several areas of the world. Likewise, the geographical distributions of several infectious diseases such as malaria, meningitis, and schistosomiasis, have been linked to intrinsic climatic and environmental factors. Research on this topic dealt with disease ecology, toxicology, pathology and/or epidemiology with regard to the emerging subject of medical geology.

- Natural Resources Damage Assessment and Management: Several environmental influences including natural and anthropogenic factors have been linked to ecosystem vulnerability. Monitoring and assessment data are therefore needed for sciencebased decision-making with regard to environmental management. Papers for presentation on this topic included those related to: a) conceptual modeling for ecological risk assessment, b) assessment of the physical, chemical, and biological characteristics of specific ecosystems, c) applications of GIS and remote sensing technology to environmental assessment and management, and d) bioindicators for environmental management.

The symposium attracted 293 participants from 21 countries representing all five continents, and 186 scientific presentations across the disciplines of environmental health and biomedical sciences. As stated above, the scientific program was composed of six plenary sessions where oral/platform presentations were given by more than 40 invited speakers. In addition, there were two poster sessions - one for faculty and professional scientists, and one for students - with more than 100 abstracts. The submitted full length manuscripts were peer-reviewed, and selected for publication by experts in their respective fields. The accepted papers are being published as special issues of the International Journal of Environmental Research and Public Health.

We wish to extend special thanks to Dr. Sidney A. McNairy, Jr., Associate Director of the NIH National Center for Research Resources and Director of the Division of Research Infrastructure, our Distinguished Symposium Banquet Speaker who pointed out that the RCMI Program at NIH has been a proud sponsor of this conference series since its inception in 2004. He echoed that "the research highlighted in the Fourth International Symposium on Recent Advances in Environmental Health Research exemplifies the Program's continuing commitment to environmental health research and the importance of global collaborations that will expedite the translation of research from the bench to the bedside and ultimately improve the health of every member of our society". Dr. Kenneth Olden, former Director of the National Institute of Environmental Health Sciences, served as Distinguished Speaker for the Biomedical Sciences and Health Information Lecture Series that is held in conjunction with the Symposium. He spoke very eloquently on the subject of gene-environment interaction and the critical role it plays in human health. Other platform presentations and keynote addresses were made 
by prominent biomedical and environmental health scientists with research expertise in cancer, diabetes, HIV/ AIDS, infectious and parasitic diseases, cardiovascular diseases, neurodegenerative diseases, gene-environment interactions, emerging technologies, health disparities and other environmentally-related illnesses. These important health issues were associated with the symposium topics.

Our appreciations are extended to Mrs. Rose Foster and Mrs. Wilma Templin-Branner of the Oak Ridge Institute for Science and Education for their valuable help with the organization of the pre-symposium workshop on the National Library of Medicine Web-Based Resources for Environmental Health and Biomedical Research. Major emphasis of the workshop was training participants on how to access and retrieve important environmental health and biomedical research information from the Toxicology Network database and other relevant web-based biomedical resources.

Special thanks are extended Dr. Ronald Mason, Jr. (JSU
President), Dr. Velvelyn Foster (Vice-President for Academic Affairs and Student Life), Dr. Felix Okojie (Vice President for Research Development, and Federal Relations) and Dr. Mary Myles (Director of Title III Program) for their administrative support. We would like to acknowledge the authors for their involvement and cooperation, and for their outstanding contributions to advancing science and sound decision making in the critical area of environmental health sciences. Special thanks are also extended to all the peer-reviewers who took time off their busy schedules to carefully and critically review each of the manuscripts.

On behalf of the entire organizing committee, the greatest acknowledgments go to our major symposium sponsors including the U.S. Department of Education Title IIIStrengthening the Environmental Science Ph.D. Program at JSU, NIH RCMI-Center for Environmental Health, Diwan University College of Management in Taiwan, JSU Office of Academic Affairs, and JSU Office of Research Development and Federal Relations. 